\section{A Importância dos Controles Domiciliares na Redução de Internações em Portadores de Diabetes Mellitus do Tipo 1}

\section{RESUMO}

Vários esquemas terapêuticos, geralmente com base na determinação da glicemia capilar, têm sido utilizados para melhorar o controle do diabetes mellitus do tipo 1 (DM1). Em vista da dificuldade de obtenção de múltiplas amostras sangüíneas diárias, pelo stress da punção e/ou pelo alto custo da determinação glicêmica, freqüentemente utilizamos a glicosúria como um parâmetro da glicemia. Em 1990 criamos um ambulatório específico para os diabéticos em nosso serviço e, desde 1992. com a doação de tiras para determinação domiciliar de glicemia, glicosúria e cetonúria aos pacientes carentes, passamos a cobrar o controle domiciliar. O objetivo do presente trabalho foi avaliar a influência da realização de controles domiciliares rotineiros no número de internações dos pacientes portadores de DM1. Analisamos os números: de pacientes acompanhados (P), de internações (I), de tiras distribuídas anualmente $(U=$ glicosúria/cetonúria, $S=$ glicemia) e a relação I/P. Observamos que apesar do aumento progressivo do número de pacientes acompanhados, houve queda das internações acompanhando o aumento progressivo do material fornecido para controle domiciliar, chegando a ser 6 a 8 vezes menor que as observadas em 1989. Houve correlação inversa entre o número de tiras urinárias fornecidas $(U / P)$ e a porcentagem de internações $(r=-0,83, p<$ 0,05 ). Concluímos que um grupo estruturado para tratamento de DM1 permitiu um melhor controle da doença com diminuição das internações e que apesar de pouco valorizada pela literatura, a determinação de glicosúria é importante no tratamento do DM 1 na faixa etária pediátrica. (Arq Bras Endocrinol Metab 2000;44/3: 215-9)

Unitermos: Diabetes mellitus; Crianças diabéticas; Monitorização domiciliar.

\section{ABSTRACT}

Several therapeutic strategies have been used to improve control of patients with type 1 diabetes mellitus 1 (DM1), most of them based on capillary glycemia determinations. Considering the difficulty to have frequent capillary blood letting due to stress and the high cost of reagent strips for home glycemia determinations, we have often used glycosuria as a parameter of glycemia. In 1990 a group was established in our service to treat DM 1 patients. Since 1992 all patients have been asked for home monitoring glycemia and glycosuria/ ketonuria. Free reagent strips were given to destitute patients. The aim of this work was to evaluate the benefits of home monitoring. We have correlated the outpatient number $(P)$; the number of hospital admittances (I); the number of blood $(S)$ and urine $(U)$ reagent strips granted yearly; and the ratio for hospital admittances/followed up patients $(I / P)$. We observed that, besides the increase in the number of patients in regular attendance, there was a decrease of 6 to 8 -fold in hospital

\section{artigo original}

\author{
Sofia Helena V. Lemos- \\ Marini \\ Maria Cristina de Lima \\ Gil Guerra Jv. \\ Maria Fernanda V.M. \\ Paulino \\ Walter J. Minicucci
}

\begin{abstract}
Ambulatório de Diabetes Pediatria Departamento de Pediatria - Hospital de Clínicas da Faculdade de Ciências Médicas da Universidade Estadual de Campinas - CIPED/UNICAMP Campinas, $S P$.
\end{abstract}

Recebido em 22/03/99 Revisado em 19/01/00 Aceito em 31/03/00 
admittances compared to the same parameters obtained during 1989. These results are accompanied by an increase on the number of reagent strips distributed. An inverse correlation ( $r=-0.83 ; p<0.05$ ) between the number of urinary reagent strips distributed and the percentage of hospital admittances was found. Our results indicate that despite the low importance the literature gives to the urine test it can be considered as an important tool for controlling DMI pediatric patients. (Arq Bras Endocrinol Metab 2000;44/3: 215-9)

Keywords: Diabetes mellitus; Diabetic children; Home-monitoring.

$\mathrm{D}$ ESDE 1993, QUANDO os resultados de estudo multicêntrico americano demonstraram que a melhora do controle do diabetes mellitus do tipo 1 (DMl) levava a menor incidência ou a atraso na progressão das complicações crônicas $(1,2)$, tenta-se, cada vez mais, manter o paciente euglicêmico para prevenção destas complicações (3-5). Para melhorar o controle da doença, a maioria dos serviços utiliza esquema de várias aplicações de misturas de insulinas de ação rápida $\mathrm{c}$ intermediária por dia; geralmente este esquema baseiase em múltiplas determinações diárias de glicemia para nortear a necessidade de insulina exógena $(6,7)$.

$O$ alto custo das tiras reagentes para determinação de glicemia (média de US $\$ 1,00$ cada tira) torna inviável a pacientes de baixo poder econômico manter este tipo de controle domiciliar.

Em pacientes da faixa etária pediátrica temos, freqüentemente, dificuldade em obter 4 a 6 amostras de sangue diárias, por falta de locais adequados para punção a longo prazo. Além disso, tal procedimento muitas vezes causa stress psicológico, o que dificulta ainda mais a determinação de múltiplas glicemias diárias. No entanto, como a manutenção da glicemia próxima aos níveis normais leva a um aumento na incidência de hipoglicemias $(8,9)$, o que pode levar a alterações neurológicas $(10,11)$ e a lesões do SNC $(12,13)$, é necessário que tenhamos, diariamente, uma estimativa dos níveis de glicemia nos nossos pacientes.

Devido às dificuldades citadas acima, utilizamos, também, a determinação da glicosúria como um parâmetro da glicemia, pois é muito mais fácil a coleta do material, e o custo das tiras urinárias chega a ser $1 / 5$ do custo das tiras para análise sanguínea.

Desde o início do ano de 1992 a Secretaria Estadual de Saúde do Estado de São Paulo tem fornecido tiras reagentes para pacientes atendidos no Ambulatório de Diabetes Pediatria do Hospital das
Clínicas da Universidade Estadual de Campinas, o que tem facilitado a realização dos controles domiciliares e nos levou a realizar este estudo sobre a sua influência no tratamento do DMI.

\section{MATERIAL E MÉTODOS}

Desde agosto de 1990, com o treinamento de enfermeiras do ambulatório geral de Pediatria, criamos uma equipe fixa, constituída por médicos e enfermeiras, responsável pelo tratamento e orientação dos pacientes com DMl.

Desde o início de 1992, época em que a Secretaria Estadual de Saúde passou a nos fornecer tiras reagentes para determinação de glicose e cetona na urina e para determinação, através de leitura visual, da glicemia, temos tentado distribuir para cada paciente carente, material para 100 controles urinários (orientamos os pacientes para divisão ao meio das tiras para glicosúria) e 25 sangüíneos mensais. Devem ser realizadas 2 a 4 determinações de glicose e cetona na urina, diariamente, e 3 a 6 de glicemia, semanalmente. Isto nem sempre tem sido possível devido à inconstância do recebimento, já que tal doação só passou a ser orçamentada no fim do ano de 1997, e à dificuldade de algumas famílias na determinação da glicemia.

A equipe de enfermagem é responsável pela orientação do uso das tiras e pela distribuição das mesmas aos pacientes. Procuramos fornecer, mensalmente, material para os controles durante 1 mês e esta doação fica registrada em livro com assinatura do paciente ou responsável, o que nos possibilitou a realização deste levantamento.

O paciente recebe, também, um caderno para marcação dos controles e das intercorrências diárias. Este caderno deve ser levado às consultas para servir de base aos médicos na orientação do tratamento e para possibilitar nova retirada de material. $O$ intervalo médio entre as consultas é de 3 a 4 meses para os pacientes com mais de um ano de doença e todos são rotineiramente orientados para procurar o serviço médico do HC/UNICAMP nas situações de urgência.

\section{COLETA DOS DADOS}

Para definir o número de pacientes portadores de DMl acompanhados em nosso ambulatório, consultamos nossos cadastros, com registro de todos os pacientes diabéticos atendidos pelo Ambulatório de Pediatria desde 1983 e, para definir a data da última consulta em nosso serviço, utilizamos os dados dos prontuários do $\mathrm{HC}$ /UNICAMP. 
Para verificar se houve mudanças no controle dos pacientes portadores de DMl, fizemos um levantamento de todas as internações por cetoacidose diabética e hipoglicemia de pacientes portadores de DM nas enfermarias do HC/UNICAMP (alguns pacientes adolescentes já são internados nas enfermarias de clínica médica), no período compreendido entre 01 de janeiro de 1989 a 31 de dezembro de 1997, e selecionamos os pacientes acompanhados em nosso ambulatório.

Utilizamos os livros de registro para definir o número de tiras distribuídas de 10 de abril de 1992 (data da primeira distribuição) a 31 de dezembro de 1997.

Os dados analisados neste trabalho referem-se ao intervalo de tempo entre 1989 e 1997, período que subdividimos em 3 fases:

1 - corresponde ao ano de 1989, quando o paciente diabético era acompanhado no ambulatório geral de pediatria;

2 - corresponde aos anos de 1990 a 1991, quando o paciente diabético passou a ser atendido por equipe fixa e treinada de médicos e enfermeiras no ambulatório de diabetes pediatria;

3 - corresponde aos anos de 1992 a 1997, época em que passamos a doar material para controle domiciliar aos pacientes carentes e a cobrar a realização destes controles de todos os pacientes do ambulatório de diabetes pediatria.

\section{METODOLOGIA ESTATÍSTICA}

Como se refere à análise de dados intervalares, utilizamos o coeficiente de correlação linear de Pearson (r) com significância (p) de 5\%.

\section{RESULTADOS}

Os resultados deste levantamento, divididos em períodos de 1 ano, encontram-se na tabela 1 e nos gráficos 1 e 2 .

Considerando os dados da tabela e dos gráficos, vemos que houve diminuição da proporção de internações, em relação ao número de pacientes acompanhados, em 1990 e 1991 quando comparados a 1989; esta queda se acentua a partir de 1992 até 1994, ano em que foi 8 vezes menor que a de 1989; nos anos de 1995 e l'996 houve aumento relativo do número de internações e em 1997 há novamente queda deste número.

Utilizando o teste de Pearson encontramos uma correlação inversa entre o número de tiras para determi-
Tabela 1 - Pacientes acompanhados, internações, tiras fornecidas, e relação entre internações e paclentes acompanhados.

\begin{tabular}{cccccc}
\hline ANO & $\mathbf{P}$ & $\mathbf{I}$ & $\mathbf{S}$ & $\mathbf{U}$ & $\mathbf{I} / \mathbf{P}$ \\
\hline 1989 & 39 & 22 & - & - & 0,56 \\
1990 & 58 & 27 & - & - & 0,46 \\
1991 & 80 & 30 & - & - & 0,37 \\
1992 & 109 & 17 & 775 & 4.800 & 0,15 \\
1993 & 129 & 12 & 2.519 & 48.125 & 0,09 \\
1994 & 146 & 11 & 4.441 & 38.815 & 0,07 \\
1995 & 170 & 24 & 3.038 & 15.090 & 0,14 \\
1996 & 186 & 26 & 11.200 & 5.760 & 0,13 \\
1997 & 187 & 18 & 12.775 & 35.850 & 0,09 \\
\hline
\end{tabular}

P: número de pacientes portadores de DM1 em acompanhamento ambulatorial; I: número de internações de pacientes portadores de DM1; S: número de tiras reagentes para determinação de glicemia fornecidas; U: número de tiras reagentes para determinação de glicosúria/cetonúria fornecidas; I / P: relação $n^{\circ}$ de internações $/ n^{\circ}$ de pacientes acompanhados

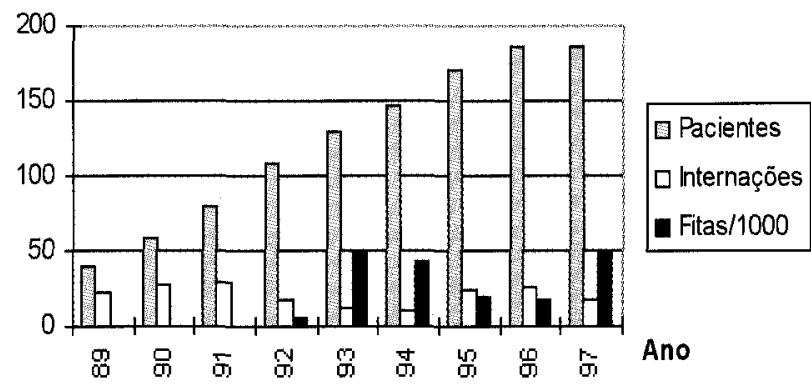

Obs.: a coluna fitas/1.000 corresponde à soma dos dois tipos de tiras, para urina e sangue distribuídas, dividida por 1.000 para melhor visualização.

Gráfico 1 - Número de pacientes acompanhados, número de internações e número total de tiras fornecidas.

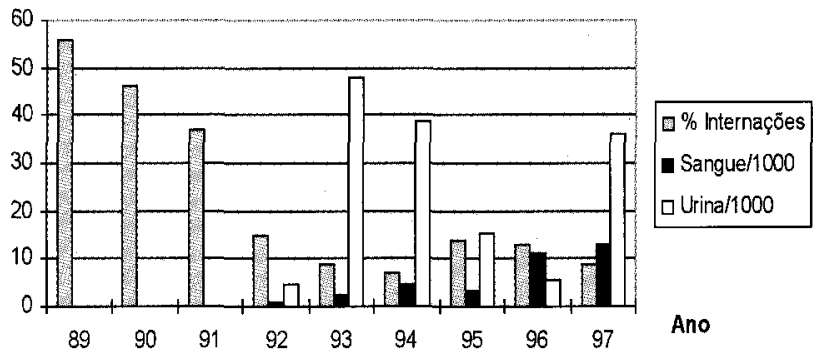

Obs:: as colunas urina/ 1.000 e sangue/ 1.000 correspondem, respectivamente, ao número de tiras para glicosúria/ cetonúria e ao de tiras para glicemia doadas, divididos por 1.000, para melhor visualização.

Gráfico 2 - Porcentagem de pacientes internados e número de tiras para glicosúria/cetonúria e para glicemia fornecidas. 
nação de glicosúria e cetonúria fornecidas e o número de internações $(r=-0,83, p=0,037)$. Não houve correlação entre o número de tiras para glicemia distribuídas e o número de internações $(r=-0,32, p=0,53)$.

\section{DISCUSSĀO}

Desde abril de 1992, a Secretaria Estadual de Saúde do Estado de São Paulo passou a fornecer em caráter especial e de maneira não contínua, material para uso domiciliar a pacientes atendidos no Ambulatório de Diabetes Pediatria do Hospital das Clínicas da UNICAMP. Tais doações consistiam de seringas para aplicação de insulina e tiras reagentes para determinação de glicemia e glicosúria/cetonúria e visavam contribuir para uma melhora do tratamento, já que a maioria de nossos pacientes não possui condições financeiras para arcar com esta despesa.

Em fevereiro de 1997 realizamos um estudo inicial analisando o número de pacientes portadores de DM1 acompanhados em nosso serviço, o número de internações anuais destes pacientes em nosso hospital e o material para controle domiciliar distribuído; os resultados levaram a Direção Regional de Saúde de Campinas - DIR XII - a orçamentar este material.

Avaliando os dados de 1989 a 1991 vemos que a formação de um grupo estruturado para acompanhamento dos pacientes portadores de DMl contribuiu para um melhor controle e, portanto, para a diminuição relativa do número de internações.

Os dados obtidos após o início da distribuição de tiras nos mostram que a queda no número de internações acentua-se quando há estímulo e se fornece meios para a realização habitual dos controles domiciliares.

Além disto, observando a correlação inversa entre o número de tiras urinárias fornecidas e a porcentagem de internações encontrada, vemos que a determinação de glicosúria e cetonúria, apesar de pouco valorizada pela literatura, foi importante no acompanhamento dos nossos pacientes. Acreditamos não ter encontrado o mesmo tipo de correlação quando analisamos as tiras para glicemia devido ao baixo número de tiras fornecido além do fato de que nem todos os pacientes ou familiares aprenderam a realizar adequadamente a determinação através de leitura visual da glicemia.

Em nossa opinião estes resultados não serão conseguidos somente através da distribuição do material. Junto a isto deve haver a educação do paciente e da família para o uso adequado do material, a cobrança da realização dos testes domiciliares e sua utilização, pela equipe médica, para nortear o ajuste do tratamento.
Concluindo, acreditamos que os resultados deste trabalho demonstram a necessidade de investir no tratamento ambulatorial do paciente diabético, criando grupos para acompanhamento e fornecendo material para tratamento e controle domiciliar, o que diminuiria o custo da doença, não só através da redução do número de internações, como também pela melhora do prognóstico pelo melhor controle da doença.

\section{AGRADECIMENTOS}

Os autores agradecem ao Prof. Aquiles Piedrabuena o estudo estatístico, ao Prof. André Moreno Morcillo o apoio técnico e à Profa. Maria Tereza Matias Baptista, a revisão do manuscrito e suas colocações, sempre oportunas, colaborando para a clareza do texto.

\section{REFERÊNCIAS}

1. DCCT Research Group. The effect of intensive treatment of diabetes on the development and progression of long-term complications in insulin-dependent diabetes mellitus. N Eng J Med 1993;329:977-86.

2. DCCT Research Group. Effect of intensive diabetes treatment on the development and progression of longterm complications in adolescents with insulin-dependent diabetes mellitus: Diabetes Control and Complications Trial. J Pediatr 1994:125:177-88.

3. American Diabetes Association. Standards of medical care for patients with diabetes mellitus. Diabetes Care 1996:19(Suppl. 1):4-11.

4. Plotnick L, Henderson R. Insulin. In: Plotnick L, Henderson $R$, eds. Clinical Management of the Child and Teenager with Diabetes. Baltimore: Johns Hopkins Unlversity Press, 1998:77-97.

5. American Diabetes Association. Routine management: objectives. Medical Management of Type 1 Diabetes. Alexandria: American Diabetes Association, 1998:24-33.

6. Daneman D. Intensive diabetes management: the pediatric perspective. International Symposium on a Current Review of Pediatric Endocrinology, 1997:13-21.

7. American Diabetes Association. Insulin administration. Diabetes Care 1996; 19(Suppl.1):20-3.

8. DCCT Research Group. Adverse events and their association with treatment regimens in the diabetes control and complications trial. Diabetes Care 1995;18: 1515-27.

9. DCCT Research Group. Epldemiology of severe hypoglycemia in the diabetes control and complications trial. Am J Med 1991:90:450-9.

10. Shehadeh N, Kassem J, Tchaban I, Ravid S, Shahar E, Naveh $\mathrm{T}$, ef al. High incidence of hypoglycemic episodes with neurologic manifestations in children with 
insulin dependent diabetes mellitus. J Pediatr Endocrinol Metab 1998;11:183-7.

11. Ryan C, Atchison J, Puczynski S, Puczynski M, Arslanian S, Becker D. Mild hypoglycemia associated with deterioration of mental efficiency in children with insulin dependent diabetes mellitus. J Pediatr 1990;1 17:32-8.

12. Soltéz $G$, Acsádi $G$. Association between diabetes, severe hypoglycemia and electroencephalographic abnormalities. Arch Dis Child 1989;64:992-6.

13. Perros P, Deary IJ, Sellar RJ, Best JJK, Frier BM. Brain abnormalities demonstrated by magnetic resonance imaging in adult IDDM patients with and without a histo- ry of recurrent severe hypoglycemia. Diabetes Care 1997;20: 1013-8.

\section{Endereço para correspondência:}

Sofia Helena Valente de Lemos-Marini

Departamento de Pediatria / CIPED

Faculdade de Clências Médicas da UNICAMP

Cidade Universitária Zeferino Vaz

Rua Alexandre Fleming, 81

Caixa Postal 6.111

13092-340 Campinas, SP 